\title{
Sulfamerazine Sodium
}

National Cancer Institute

\section{Source}

National Cancer Institute. Sulfamerazine Sodium. NCI Thesaurus. Code C80794.

A sodium salt form of sulfamerazine, a long-acting sulfanilamide antibacterial agent. 